\title{
Erratum to: Guidelines on the management of IgE-mediated food allergies
}

Erratum to: Allergo J Int 2015;24:256-93

DOI: 10.1007/s40629-015-0074-0

In the original publication of the article, table 8 and table 9 were missing some allergens which are now available for in vitro diagnosis. The revised and updated tables are presented below.
Unfortunately, author Alexander Nast was omitted in the original publication of the article.

Additionally, the Digital Object Identifier (DOI) of the original article was incorrect. The correct DOI is $10.1007 / \mathrm{s} 40629-015-0074-0$.

The online version of the original article can be found under http://dx.doi.org/10.1007/s40629-015-0074-0
German version

www.springer-

medizin.de/

allergo-journal

Tab. 8: Selected food allergens and their sources of plant origin ${ }^{a, b}$

Protein families

Storage proteins (protein families, structure)

\begin{tabular}{l|l|l|l|l|l|l|}
\hline & & & \multicolumn{2}{l}{ Storage proteins (protein families, structure) } \\
\hline $\begin{array}{l}\text { Bet-v-1 } \\
\text { homologs }\end{array}$ & LTP & Profilins & Thaumatins & Oleosins & Prolamins & Cupins \\
\hline & & & & & $\begin{array}{l}7 / 85 \text { Globumins } \\
\text { (vicilin) }\end{array}$ & $\begin{array}{l}115 \text { Globulin- } \\
\text { (legumin) }\end{array}$ \\
\hline
\end{tabular}

Apple

Mal d 1

Mal d 3

Mal d 4

Mal d 2

Peanut

Ara h 8

Ara h 9

Ara h 5

Ara h $10(16 \mathrm{kD})$

Ara h $11(14 \mathrm{kD})$

Ara h 2

Ara h 1

Arah 3

Ara $\mathrm{h} 7$

Spices

Bell

pepper

Parsley

Pet c 1

Pet $c 3$

Cap a 2

Pet c 2

Hazelnut

Cor a 1

Cor a 8

Cor a 2

Cor a 12 (17 kD)

Cor a 14

Cor a 11

Cor a 9

Cor a $13(14 / 16$

$\mathrm{kD})$

\begin{tabular}{|l|l|l|l|}
\hline Carrot & Dau c 1 & Dau c 3 & Dau c 4 \\
\hline Cherry & Pru av 1 & Pruav 3 & Pru av 4 \\
\hline Kiwi & Act d 8 & & Act d 9 \\
\hline Peach & Pru p 1 & Pru p 3 & Pru p 4 \\
\hline Celery & Api g 1 & & Api g 4 \\
\hline Sesame & & & \\
\hline Soybean & Gly m 4 & Gly m 1 & Gly m 3 \\
\hline Wheat & & Tri a 14 & Tri a 12 \\
\hline
\end{tabular}

Pru av 2

Tri a $14 \quad$ Tri a 12

\begin{tabular}{|c|c|c|}
\hline $\begin{array}{l}\text { Ses i } 4 \\
\text { Sesi } 5\end{array}$ & Sesi 1 & Ses i \\
\hline
\end{tabular}

Gly $m 5$

Gly $\mathrm{m} 6$

Tri a 19

( $\omega$-5-gliadin) 


\begin{tabular}{|c|c|c|c|c|}
\hline & \multicolumn{4}{|l|}{ Protein families } \\
\hline & Parvalbumins & Tropomyosins & Lysozymes/a-lactalbumins & Other proteins (various families) \\
\hline Hen's egg & & & Gal d 4 (lysozyme C) & $\begin{array}{l}\text { Gal d } \mathbf{1} \text { (ovomucoid, trypsin inhibitor) } \\
\text { Gal d } \mathbf{2} \text { (ovalbumin, serpin) } \\
\text { Gal d } \mathbf{3} \text { (ovotransferrin, conalbumin) }\end{array}$ \\
\hline Fish & $\begin{array}{l}\text { Gad c } 1 \\
\text { Cyp c } 1\end{array}$ & Ani s $3^{b}$ & & \\
\hline $\begin{array}{l}\text { Crustaceans/ } \\
\text { molluscs }\end{array}$ & Hom a 6 & $\begin{array}{l}\text { Cha f } 1 \\
\text { Hom a } 1 \\
\text { Met e } 1 \\
\text { Pen a } 1\end{array}$ & & \\
\hline Cow's milk & & & Bos d 4 (a-lactalbumin) & $\begin{array}{l}\text { Bos d } 5 \text { ( } \beta \text {-lactoglobulin, lipocalin) } \\
\text { Bos d } 6 \text { (bovine serum albumin) } \\
\text { Bos d } 8 \text { (casein) }\end{array}$ \\
\hline
\end{tabular}

Prof. Dr. Margitta Worm

Allergy-Center-Charité

Department of Dermatology and Allergy

Charité - Universitätsmedizin Berlin

Charitéplatz 1

10117 Berlin, Germany

E-Mail: margitta.worm@charite.de

\section{Cite this as:}

Worm M, Reese I, Ballmer-Weber B, Beyer K, Bischoff SC, Claßen M, Fischer PJ, Fuchs T, Huttegger I, Jappe U, Klimek L, Koletzko B, Lange L, Lepp U, Mahler V, Nast A, Niggemann B, Rabe U, Raithel M, Saloga J, Schäfer C, Schnadt S, Schreiber J, Szépfalusi Z, Treudler R, Wagenmann M, Watzl B, Werfel T, Zuberbier T, Kleine-Tebbe J. Erratum to: Guidelines on the management of IgE-mediated food allergies. S2k-Guidelines of the German Society for Allergo- logy and Clinical Immunology (DGAKI) in collaboration with the German Medical Association of Allergologists (AeDA), the German Professional Association of Pediatricians (BVKJ), the German Allergy and Asthma Association (DAAB), German Dermatological Society (DDG), the German Society for Nutrition (DGE), the German Society for Gastroenterology, Digestive and Metabolic Diseases (DGVS), the German Society for Oto-Rhino-Laryngology, Head and Neck Surgery, the German Society for Pediatric and Adolescent Medicine (DGKJ), the German Society for Pediatric Allergology and Environmental Medicine (GPA), the German Society for Pneumology (DGP), the German Society for Pediatric Gastroenterology and Nutrition (GPGE), German Contact Allergy Group (DKG), the Austrian Society for Allergology and Immunology (ÖGAI), German Professional Association of Nutritional Sciences (VDOE) and the Association of the Scientific Medical Societies Germany (AWMF). Allergo J Int 2015;24:333-4

DOI: $10.1007 / \mathrm{s} 40629-015-0075-z$ 